\title{
Measuring the Effect of Packet Corruption Ratio on Quality of Experience (QoE) in Video Streaming
}

\author{
Jonah Joshua $^{1}$, Akpovi Ominike ${ }^{2}$, Oludele Awodele ${ }^{3}$, Achimba Ogbonna ${ }^{4}$ \\ Computer Science Department, Babcock University, Ogun State, Nigeria
}

\begin{abstract}
The volume of Internet video traffic which consists of downloaded or streamed video from the Internet is projected to increase from 42,029PB monthly in 2016 to $159,161 \mathrm{~PB}$ monthly, in 2021, representing a $31 \%$ increase in the Compound Annual Growth Rate (CAGR). The market for mobile network operators is unpredictable, fast-paced and very competitive. End users now have more options when choosing service providers. With superior network Quality of Experience (QoE), service providers can increase margins by charging more for better quality. Packet corruption occurs when the receiver cannot correctly decode transmitted bits. This study identified the threshold at which the QoE of video streaming services became unacceptable due to the effect of packet corruption. In this paper, several experiments were carried out on video streaming services, creating disturbances to evaluate the user satisfaction level using the mean opinion scores. Network Emulator (NetEm) tool was used to create the packet corruption experienced during the video sessions and the QoE for different packet corruption percentages was established. From the experiments conducted, we found that user QoE decreased as the Packet Corruption Ratio (PCR) increased. With knowledge of the effect of the PCR, service providers can ensure that the PCR is kept within acceptable limits from end-to-end and this will ultimately lead to superior QoE from end users, which will in turn translate to improved subscriber base and profitability.
\end{abstract}

Keywords-Network Emulator; Packet Corruption Ratio (PCR); Quality of Experience (QoE)

\section{INTRODUCTION}

The growth forecast for the email, data traffic and web category, which comprises file transfers, instant messaging, web browsing, email and other Internet applications, is expected to increase from $9,059 \mathrm{~PB}$ to $19,538 \mathrm{~PB}$ per month, in the period from $2016-2021$, representing a $17 \%$ growth in the CAGR. This category contains internet traffic produced by all Internet users. Similarly, in the internet video category which consists of downloaded or streamed video from the Internet, the volume of traffic is projected to increase from $42,029 \mathrm{~PB}$ to $159,161 \mathrm{~PB}$ monthly, representing a $31 \%$ increase in the CAGR [1]. Fig. 1 shows the expected projection for consumer web, email, data and Internet video traffic.

Presently, over twenty five percent (25\%) of the global population uses advanced mobile phones (with numerous functionalities) and other smart devices to access the internet. The web has turned into a fundamental piece of our regular daily existence: Social, business, education and health amongst others. If service providers understand the QoE of popular services, it would improve their long term profitability and also prevent customers churning as a result of inferior
QoE [2]. The broad area of Quality of Experience (QoE) is gaining momentum in networks and telecommunications today, but QoE is not an entirely new concept [3]. Quality of Experience (QoE) is defined as "how a user perceives the usability of a service when in use-how satisfied he or she is with a service in terms of, for example, usability, accessibility, retainability and integrity of the service" [4]. It is an estimation used to decide how well a system is fulfilling the users' needs. The concept of QoE is important as it expresses people's perceived value of services. It can be defined in various ways. For example, communication service providers express the concept of user experience as QoE while researchers in Human-Computer interaction (HCI) characterize this concept as User Experience (UX).

"Packet corruption occurs when the receiver cannot correctly decode transmitted bits. Such decoding errors cause the cyclic redundancy check in the Ethernet frame to fail and force the receiver to drop the packet" [5]. Most communication networks experience significant fluctuations in transmission link performance over time. Corrupt packets are normally discarded by the receiver and the information that was sent originally from the source is lost and must be resent. This process of sending and receiving the packets and retransmitting corrupt packets is a costly one for the network operators [6]. It has been established that packet corruption impacts fewer links but can be more severe than congestion. Also, packet corruption rate does not correlate with utilization and link location. Some of the main causes of corruption are connector contamination, damaged or bent fiber optic cables and bad or loose transceivers [5].

In this paper, subjective experiments were carried out in a dedicated laboratory for subjective analysis. A Network testbed was setup in the laboratory to simulate the dynamic behavior of a normal wide Area Network (WAN) using an Ubuntu Linux 18.04 server and a Network Emulator (NetEm) tool. The NetEm tool was used to create artificial packet corruption by introducing single bit errors at a random offset in the packet. This emulates random noise, and can be used to emulate noisy links, such as wireless links.

We conducted experiments with different packet corruption rate percentages and different video content types such as news videos, streaming services like Netflix, YouTube and Footytube. The effect of the disturbances introduced was used to evaluate the user's Quality of Experience. The experiments followed the ITU-T stipulated guidelines for QoE subjective studies [7]. 


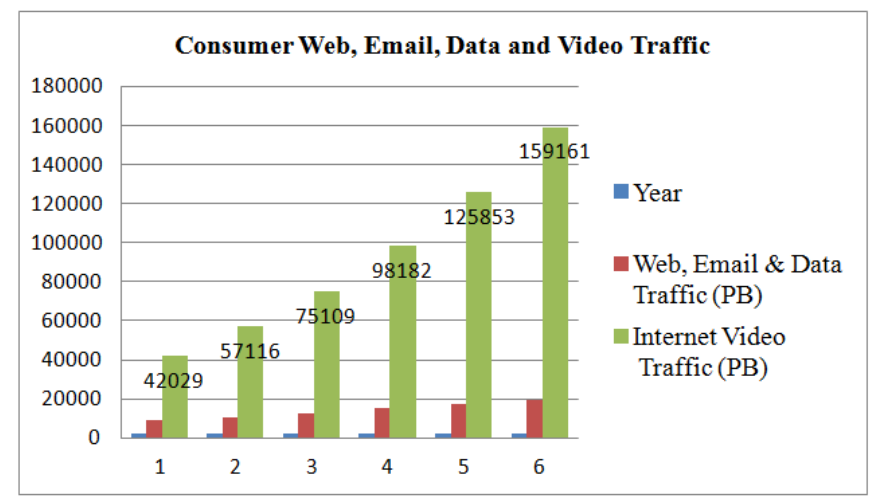

Fig. 1. Consumer Web, Email, Data and Video Traffic.

\section{REVIEW OF RELATED WORKS}

Obtaining a rich QoE is possible if service providers can properly keep an eye on how the services and applications are performing from the end-user's perspective. Research has shown that the utilization and acceptance of multimedia services is constantly growing and this provides a massive revenue generation opportunity for operators and service providers. The authors in [8] proposed a video streaming QoE assessment model to evaluate the QoE of video services by utilizing certain network layer QoS metrics. They deployed QoS parameters because network performance ensures and guarantees reliable and efficient delivery of real-time video streaming services. Based on their model, network providers can predict satisfactory QoE for end users.

In [6], the authors analyzed packet corruption pattern from data collected from a sensor network over a period of one year. In this analysis, they considered independent transmission errors and proposed a method to recover information about the original content of a corrupt packet. In [9], the authors investigated challenges with large-scale live video delivery. They achieved this by studying live video delivery that was streamed by over 600 thousand viewers in the United States of America. In analyzing the QoE for the event, they noted that a significant number of users suffered QoE impairments and thus showed lower engagement metrics.

Experiments were conducted by [10] to measure the effect of packet reordering on $\mathrm{QoE}$ in video streaming. In their paper, they achieved this using the NetEm tool and video sequences at different packet reorder percentages.

\section{QOE ASSESSMENTS AND EXPERIMENTS}

In order to investigate the QoE, an experimental Testbed on which the users were tested for specific scenarios was created. It is important to mention that for consistency, the scenarios were the same for all the users that were tested during the investigation. The experiments were performed in a dedicated laboratory. The procedures for setting up and carrying out the experiments followed the ITU-T recommendations for QoE subjective studies [7].

The subjective assessment directly measured the quality of end user experience by asking human assessors to rate the quality of the video services and applications being investigated. The key benefit of conducting laboratory studies is that the experimenter has full control over the overall evaluation process, including the context and content. In addition to that, those involved in the experiments were directly briefed and observed on the spot, thereby providing methodological and substantive results [11]. The pseudocode for the QoE estimation process is described in Fig. 2 and the As-Built diagram for the experiment setup is shown in Fig. 3.

The NetEm tool was used to create artificial packet corruption by defining rules to corrupt the packets egressing from interface enp3s0 on the Ubuntu Server. To add the traffic control rule to corrupt $10 \%$ of the packets egressing from interface enp3s 0 we used the command:

tc qdisc add dev enp3s0 root netem corrupt $10 \%$

At the end of the experiments, all the added rules were deleted using the command:

tc qdisc del dev enp3s0 root netem corrupt $10 \%$

\begin{tabular}{|c|c|}
\hline Input: & $\begin{array}{l}\text { Video Streaming Services whose QoE is to be } \\
\text { determined }\end{array}$ \\
\hline Output: & QoE MOS rankings \& User satisfaction level \\
\hline & $\begin{array}{l}\text { Step 1: Emulate the network for the experiment } \\
\text { Initialize netem emulator by loading the kernel } \\
\text { module } \\
\text { Emulate the Packet Corruption network condition. } \\
\text { Step 2: Initialize experiment } \\
\text { Step 3: Begin Test. Each test should last for a } \\
\text { maximum duration of } 20 \text { minutes. } \\
\text { Step 4: At end of experiment, subjects should rate their } \\
\text { experiences and provide MOS } \\
\text { Step 5: Calculate average MOS } \\
\text { Step 6: End Test. Flush all outbound conditions } \\
\text { configured in netem. }\end{array}$ \\
\hline End & \\
\hline
\end{tabular}

Fig. 2. Algorithm for QoE Estimation.

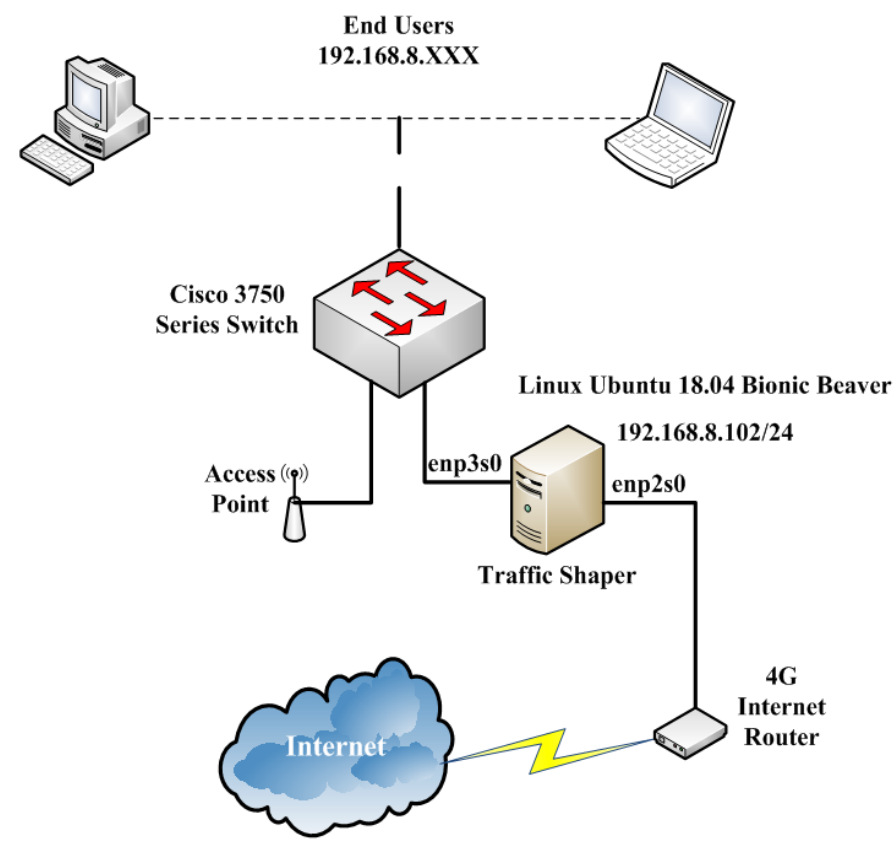

Fig. 3. As-Built Diagram for the Experiment Setup. 
TABLE I. MOS DESCRIPTORS

\begin{tabular}{|l|l|}
\hline Quality & MOS \\
\hline Excellent & 5 \\
\hline Good & 4 \\
\hline Fair & 3 \\
\hline Poor & 2 \\
\hline Bad & 1 \\
\hline
\end{tabular}

The experiments were conducted for different test scenarios and the user perceived QoE was evaluated after each test condition using the MOS (Mean Opinion Score) absolute category scale. The MOS scale has five grades, with each one reflecting users' judgment of the experiment under test. The MOS was used because a complex reporting system may result in low participation rate. Table I shows a typical MOS scale descriptor which was presented to the subjects during experiments.

\section{A. Participants and Procedure}

In this experiment, the packet corruption ratio was varied in ascending order of magnitude from 5\% to $40 \%$ with intervals of $5 \%$. The participants were unaware of the packet corruption ratio at each level. A total of 29 participants, 18 Males and 11 Females were involved in the study. The average age of the subjects was 28. There was a preexperiment briefing of 10 minutes at the start of each experiment and a 10 minute period after each experiment to debrief the users. The duration of each experiment was for 20 minutes. For the video streaming sessions, popular video streaming services like Netflix, YouTube and Footytube were used. In addition, other video content types, like news pages were used.

\section{RESULTS AND DisCUSSIONS}

Table II shows a summary of the results obtained from the experiments and Fig. 4 shows the graph of overall QoE plotted against Packet Corruption Ratio.

\section{A. Discussion}

In this study, netem creates the packet corruption by introducing a single bit error at a random offset in the packet. This emulates random noise and it may be used to emulate noisy links for example wireless links. The results obtained are shown in Table II. This study revealed that the MOS was 4.76 at PCR of 5\% and this value dropped significantly to 1.55 at $40 \%$. As the PCR increased, the MOS of users decreased correspondingly. It was observed that a negative correlation existed between the PCR and the average MOS.

TABLE II. PACKET CORRUPTION RATIO (PCR) COMPARISON

\begin{tabular}{|l|l|l|}
\hline S/N & Packet Corruption Ratio (\%) & $\begin{array}{l}\text { Average Mean Opinion Score } \\
\text { (MOS) }\end{array}$ \\
\hline 1 & 5 & 4.76 \\
\hline 2 & 10 & 4.48 \\
\hline 3 & 15 & 3.72 \\
\hline 4 & 20 & 3.52 \\
\hline 5 & 25 & 3.55 \\
\hline 6 & 30 & 2.72 \\
\hline 7 & 35 & 1.72 \\
\hline 8 & 40 & 1.55 \\
\hline
\end{tabular}

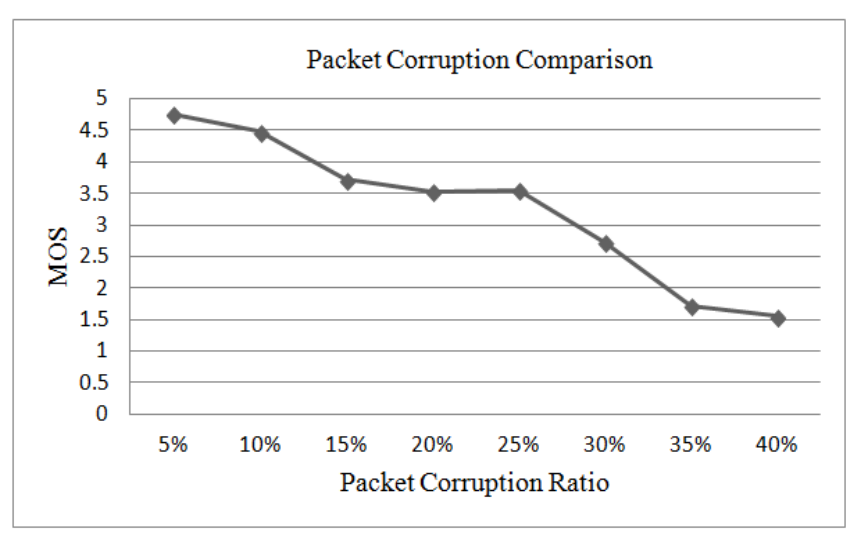

Fig. 4. MOS vs. Packet Corruption Comparison

\section{CONCLUSION}

In this research paper, several experiments were conducted on video streaming services by creating artificial packet corruption conditions by defining rules to corrupt the packets egressing from ethernet interface the Network Emulator. This was done to establish the acceptable PCR threshold value for the end users. The study showed that at PCR of $30 \%$ and above, users were no longer satisfied with the video services and were unwilling to continue using such services. With these available results, network operators can ensure that to fulfill end users' quality expectations and ensure acceptability of their service offerings, the packet corruption ratio should be kept below the acceptable thresholds presented earlier. The study was limited to investigating the effect of PCR on QoE of video streaming services. In future, we plan to examine the effect of other metrics on the QoE. There was restricted subject diversity as the experiments were conducted in a laboratory setting and majority of the participants for the tests were largely researchers and members of the academia. In future, field trials could be carried out or crowdsourcing approaches employed.

\section{REFERENCES}

[1] Cisco, "Cisco Visual Networking Index: Global Mobile Data Traffic Forecast Update, 2016-2021 White Paper," Cisco, pp. 2016-2021, 2017.

[2] P. Casas, M. Varela, P. Fiadino, M. Schiavone, H. Rivas, and R. Schatz, "On the analysis of QoE in cellular networks: From subjective tests to large-scale traffic measurements," IWCMC 2015-11th Int. Wirel. Commun. Mob. Comput. Conf., pp. 37-42, 2015.

[3] M. Fiedler and T. Minhas, "Quality of Experience Hourglass Model," IEEE, pp. 1-36, 2013.

[4] D. Soldani, M. Li, and R. Cuny, QoS and QoE Management in UMTS Cellular Systems. 2006.

[5] D. Zhuo, M. Ghobadi, R. Mahajan, K.-T. Förster, A. Krishnamurthy, and T. Anderson, "Understanding and Mitigating Packet Corruption in Data Center Networks," Proc. Conf. ACM Spec. Interes. Gr. Data Commun. - SIGCOMM '17, pp. 362-375, 2017.

[6] F. Hermans, H. Wennerström, L. McNamara, C. Rohner, and P. Gunningberg, "All is not lost: Understanding and exploiting packet corruption in outdoor sensor networks," Lect. Notes Comput. Sci. (including Subser. Lect. Notes Artif. Intell. Lect. Notes Bioinformatics), vol. 8354 LNCS, pp. 116-132, 2014.

[7] International Telecommunication Union, "ITU-T Recommendation P.1501: Subjective testing methodology for web browsing," 2014.

[8] H.-J. Kim, D.-G. Yun, H.-S. Kim, K.-S. Cho, and S.-G. Choi, "QoE assessment model for video streaming service using QoS parameters in 
wired-wireless network," Adv. Commun. Technol. (ICACT), 2012 14th Int. Conf., pp. 459-464, 2012.

[9] A. Ahmed, S. Zubair, and A. Khakpour, "QoE Analysis of a Large-Scale Live Video Streaming Event," ACM SIGMETRICS Perform. Eval. Rev., pp. 395-396, 2016.

[10] A. A. Laghari, H. He, and M. I. Channa, "Measuring Effect of Packet
Reordering on Quality of Experience (QoE) in Video Streaming," 3D Res., vol. 9, no. 3, p. 30, 2018.

[11] P. Casas, R. Schatz, F. Wamser, M. Seufert, and R. Irmer, "Exploring QoE in Cellular Networks: How Much Bandwidth do you Need for Popular Smartphone Apps ?," AllThingsCellular '15 Proc. 5th Work. All Things Cell. Oper. Appl. Challenges, no. 1, pp. 13-18, 2015. 